\title{
Cognitive Deficits in Chronic Stroke Patients: Neuropsychological Assessment, Depression, and Self-Reports
}

\author{
Arne E. Nakling a,b Dag Aarsland ${ }^{\text {b, } c}$ Halvor Næss ${ }^{a, d}$, e \\ Daniel Wollschlaeger ${ }^{f}$ Tormod Fladbyg, h Håkon Hofstad ${ }^{i}$ Eike Wehling ${ }^{i}$ \\ anstitute of Clinical Medicine, University of Bergen, Bergen, Norway; ${ }^{b}$ Centre for Age- \\ Related Medicine, Stavanger University Hospital, Stavanger, Norway; ' Institute of Psychiatry, \\ Psychology, and Neuroscience, King's College, London, UK; 'd Department of Neurology,

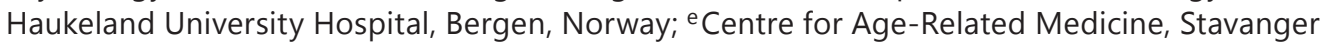 \\ University Hospital, Stavanger, Norway; ${ }^{f}$ Department of Medical Statistics, Epidemiology, and \\ Informatics, University Medical Center, Johannes Gutenberg University Mainz, Mainz, Germany; \\ ${ }^{9}$ Department of Neurology, Akershus University Hospital, Lørenskog, Norway; ${ }^{\text {h}}$ Department \\ of Neurology, Faculty Division, Akershus University Hospital, University of Oslo, Lørenskog, \\ Norway; 'Department of Physical Medicine and Rehabilitation, Haukeland University Hospital, \\ Bergen, Norway
}

\section{Keywords}

Stroke $\cdot$ Cognitive impairment $\cdot$ Stroke Impact Scale $\cdot$ Self-report $\cdot$ Depression

\section{Abstract}

Background: Following stroke, clinicians are challenged to detect and untangle symptoms of cognitive dysfunction and mood disorders. Additionally, they need to evaluate the informative value of self-reports to identify patients in need of further attendance. Aims: To examine the association between neuropsychological measures, symptoms of depression, and self-reported cognitive function. Methods: One-hundred and five chronic stroke patients underwent assessment covering 6 cognitive domains and answered the Hospital Anxiety and Depression Scale and the Memory and Thinking Scale from the Stroke Impact Scale 1 year after stroke. Age and gender difference in cognitive impairment were examined; linear regression was used to predict depression scores. Sensitivity and specificity analyses were used to validate selfreported functioning against performance on cognitive tests. Results: Cognitive impairment was observed in $60 \%$ of the patients in at least 1 cognitive domain. Cognitive performance was associated with symptoms of depression as well as with self-reported cognitive function. The final analyses revealed low sensitivity and specificity for the Memory and Thinking subscale from the Stroke Impact Scale. Conclusion: Cognitive impairment occurs frequently even in patients in a chronic phase after stroke and predicts symptoms of depression. Using the Stroke Impact Scale, clinicians should be aware of low sensitivity of self-reported cognitive function. 
Nakling et al.: Cognitive Deficits in Chronic Stroke Patients: Neuropsychological Assessment, Depression, and Self-Reports

\section{Introduction}

Stroke is the second leading cause of death and a common cause of physical and cognitive disability in patients in the developed countries $[1,2]$. The importance of stroke management after discharge is increasingly recognized to be able to reduce the burden of stroke and to ensure independence. Clinicians are challenged to appraise those patients needing attendance, in particular when considering that the population above the age of 65 years is the fastest growing group in western countries [3], and age is a major risk factor for stroke and consequent dementia [4].

Cognitive impairment emerging after stroke is an increasingly recognized factor for longterm disability. The prevalence of cognitive impairment varies across studies, depending on assessment methods, definitions, or sample characteristics. Frequently, screening instruments like the Mini-Mental State Examination (MMSE) [5] or the Montreal Cognitive Assessment (MoCa) [6] are applied even though they seem unsuitable to identify subtle or specific cognitive deficits [7]. Neuropsychological assessment reveals that deficits in executive functioning, attention, mental processing speed, visual perception, and construction ability are common ailments both in subacute and chronic patients [8-12].

The risk of proceeding cognitive decline and dementia after stroke is often stated. Yet, several authors have stressed the multiple evolutionary trends in cognitive changes in chronic stroke patients [12-14]. They found an annual conversion to dementia in 8-13\% within the first year after stroke. Furthermore, the findings indicated that a large proportion of patients $(50 \%)$ demonstrated improvement in cognitive functioning [14] or stable impairment (78\%) [13]. Two recent longitudinal studies demonstrated that stroke increases the risk of persistent and cognitive decline in particular in executive functioning $[15,16]$. One time-varying factor that may influence cognitive performance over time is the level of depressive symptoms.

Poststroke depression (PSD) is reported to occur with prevalence rates between 5 and $64 \%$, with this variation depending on study population, time of assessment after stroke, and assessment instruments $[17,18]$. PSD is known to be related to mortality, reduced functional outcome, and quality of life [19-21], and may be linked to several factors and psychological mechanisms. With regard to demographic variables and their association with depression in stroke patients, there seems to be no clear pattern. Most studies seem to reveal that higher age is not associated with depression in stroke, yet not all could support these findings [20, 22]. Additionally, and in some contrast to the general population, female stroke patients do not seem more affected by depression than males [17]. PSD has been found to be related to cognitive impairment such as attention, memory, visual perception and construction, and language $[23,24]$. Yet, the direction whether cognitive impairment leads to depression or whether PSD leads to impairment is still being debated [25, 26].

In clinical settings, self-reports on cognitive function are common in stroke management, and it has been shown that patients frequently report reduced mental tempo, memory deficits, and difficulties with concentration [27, 28]. However, it has not always been possible to confirm these complaints with empirical data [29]. Subjective cognitive complaints (SCC) have rather been shown to be associated with factors such as satisfaction with the social network, self-reported emotional difficulties, and symptoms of depression and neuroticism scores [27, 30-32]. Van Rijsbergen et al. [29] concluded in their review the frequent occurrence of SCC after stroke. Even though they could not determine a clear pattern relating SCC to objective cognitive performance or depressive symptoms, they stressed that patients with SCC should be monitored by clinicians as this may be indicative for cognitive decline.

The Stroke Impact Scale (SIS) was developed to take patients' and caregivers' perspective of impact of stroke on health and functional status into account. It includes a separate scale on memory function and thinking. So far, few studies have combined cognitive performance 
Nakling et al.: Cognitive Deficits in Chronic Stroke Patients: Neuropsychological Assessment, Depression, and Self-Reports

measures and self-reported functioning on the SIS subscale on memory and thinking (mtSIS).

The aim of the current study was to determine cognitive functioning in chronic stroke patients using comprehensive neuropsychological assessment. We also wanted to examine the association between objective cognitive performance and depression before analyzing the association between self-reported and objectively assessed cognitive function.

\section{Methods and Materials}

\section{Patients}

The patients were prospectively recruited between 2008 and 2011 at the Department of Neurology at the Haukeland University Hospital, Norway. All stroke patients coming to the unit in the relevant period were considered for inclusion, and all patients who were considered relevant were invited in person to participate by the treating neurologist. Inclusion criteria comprised that patients were home-dwelling at the time of admission, had verification of stroke by magnet resonance imaging (MRI) or computer tomography (CT), and had a National Institutes of Health Stroke Scale (NIHSS) [33] score between 2 and 26 (higher scores indicating poorer function). Patients with an NIHSS score $<2$ were included if they had a score $\geq 2$ on the modified Rankin Scale, a scale used to assess the level of dependence of daily activities (higher scores indicating more disabilities). Exclusion criteria were severe psychiatric disorder, alcohol or substance abuse, serious conditions interfering with the subsequent rehabilitation process, and insufficient knowledge of the Norwegian language (prestroke). At the initial hospital admission, patients had a comprehensive neurological examination to establish a diagnosis of stroke, clinical syndrome, pathological, and etiological subtype of stroke and neurological deficits, as assessed by the NIHSS, and MRI or CT. They had to be awake, and informed consent to participate in the project was obtained. If the patient was not capable of giving his or her consent, it could be given by a next of kin. There was no age limit. Three-hundred and twenty-two patients were originally registered. The project was approved by the Regional Committee for Research Ethics of Western Norway. The study was performed according to the Declaration of Helsinki on guidelines for biomedical research involving human subjects.

One year after initial admission, the by then all discharged patients were invited to return for a comprehensive neuropsychological assessment including a battery of cognitive tests and questionnaires. In total, $n=143$ patients returned for cognitive assessment.

Of these, $n=13$ were unable or did not wish to complete the neuropsychological assessment due to fatigue or global deficits, and $n=25$ were excluded due to aphasia. This left a sample of $n=105$ eligible for the current study $(n=98$ with cerebral infarction, $n=7$ with cerebral hemorrhage). Clinical characteristics registered during admission are presented in Tables 1 and 2.

\section{Assessment of Cognitive Function}

To assess different aspects of cognitive functioning, each participant was tested in an approximately 3-hour session. The tests were administered and scored by well-trained test technicians or psychologists. The MMSE [5], a 30-point questionnaire, was included as a screening instrument on global cognitive function. Frequently, a cutoff of $<24$ is used to define cognitive impairment [7]. Yet, knowing about the challenges to detect subtle deficits, we used a cutoff of $<26$ to define mild or more extensive cognitive deficits.

Since several of the neuropsychological tests provide several test conditions and subscores, these were combined according to standard clinical practice and manuals in order 
Table 1. Baseline characteristics of all patients according to the protocol at admission

\begin{tabular}{|c|c|c|c|c|}
\hline & $\begin{array}{l}\text { Total sample } \\
\text { upon admission } \\
(n=322)\end{array}$ & $\begin{array}{l}\text { Not } \\
\text { returners } \\
(n=179)\end{array}$ & $\begin{array}{l}\text { Not } \\
\text { assessable } \\
(n=38)\end{array}$ & $\begin{array}{l}\text { Study sample } \\
(n=105)\end{array}$ \\
\hline \multicolumn{5}{|l|}{ Registered on admission } \\
\hline Age upon admission, years ${ }^{1}$ & $71.5(14.4)$ & 75.7 (13.8) & $72.3(12.3)$ & $67.5(12.5)$ \\
\hline Education $^{1}$ & $\mathrm{nr}$ & $\mathrm{nr}$ & $10.3(3.1)$ & $6.4(5.5)$ \\
\hline NIHSS score on admission ${ }^{1}$ & $5.6(5.2)$ & $6.4(5.5)$ & $7.50(5.4)$ & $4.88(4.6)$ \\
\hline Stroke type ischemic ${ }^{2}$ & $286(89)$ & $156(87)$ & 34 (89) & $96(91)$ \\
\hline Male $^{2}$ & $178(55)$ & $93(52)$ & $24(63)$ & $61(58)$ \\
\hline Diabetes $^{2}$ & $49(15)$ & $29(16)$ & $8(21)$ & $12(11)$ \\
\hline Myocardial infarction $^{2}$ & $46(14)$ & $24(13)$ & $8(21)$ & $14(13)$ \\
\hline Angina pectoris ${ }^{2}$ & $42(14)$ & $26(15)$ & $7(18)$ & $9(9)$ \\
\hline Peripheral artery disease ${ }^{2}$ & $20(6)$ & $16(9)$ & $3(8)$ & $1(1)$ \\
\hline Leukoaraiosis $^{2}$ & $120(37)$ & $74(41)$ & $13(34)$ & $33(31)$ \\
\hline Hypertension $^{2}$ & $186(58)$ & $111(62)$ & $17(45)$ & $58(55)$ \\
\hline Earlier stroke $^{2}$ & $50(16)$ & $33(18)$ & $5(13)$ & $12(11)$ \\
\hline Earlier transient ischemic attack ${ }^{2}$ & $24(8)$ & $15(8)$ & $3(8)$ & $6(6)$ \\
\hline \multicolumn{5}{|l|}{ Follow-up assessment } \\
\hline Age $\geq 60$ years at follow-up ${ }^{2}$ & $\mathrm{nr}$ & $153(86)$ & $32(84)$ & $75(71)$ \\
\hline $\operatorname{MMSE}^{1,3}$ & $\mathrm{nr}$ & $\mathrm{nr}$ & $24.7(4.4)$ & $28.1(2.2)$ \\
\hline HADS Depression Scale ${ }^{1}$ & $\mathrm{nr}$ & $\mathrm{nr}$ & $4.5(3.1)$ & $3.1(3.2)$ \\
\hline HADS Anxiety Scale ${ }^{1}$ & $\mathrm{nr}$ & $\mathrm{nr}$ & $4.3(3.6)$ & $3.3(3.32)$ \\
\hline HADS total ${ }^{1}$ & $\mathrm{nr}$ & $\mathrm{nr}$ & $8.9(5.7)$ & $6.5(5.9)$ \\
\hline
\end{tabular}

nr, not registered; NIHSS, National Institutes of Health Stroke Scale; MMSE, Mini-Mental State Examination; HADS, Hospital Anxiety and Depression Scale. ${ }^{1}$ Values represent mean (standard deviation). ${ }^{2}$ Values represent $n(\%) .{ }^{3}$ Raw score.

Table 2. Distribution of patients $(n=98)$ with cerebral infarction according to causes (TOAST criteria) and stroke location (OCPS criteria)

\begin{tabular}{lr} 
TOAST criteria & \\
Large artery atherosclerosis & 9 \\
Cardioembolism & 26 \\
Small vessel occlusion & 23 \\
Stroke of other determined etiology & 3 \\
Stroke of undetermined etiology & 37 \\
\hline OCPS criteria & \\
Partial anterior circulation infarct (PACI) & 37 \\
Posterior circulation infarct (POCI) & 21 \\
Total anterior circulation infarct (TACI) & 6 \\
Lacunar infarct (LACI) & 34
\end{tabular}

TOAST, Trial of Org 10172 in Acute Stroke Treatment; OCPS, Oxfordshire Community Stroke Project Criteria.

to define 6 cognitive domains. Verbal memory was assessed with the California Verbal Learning Test (CVLT II) [34]. The subscores combined for the verbal memory domain were performance scores on total learning, short delay free recall, and long delay free recall. Executive functioning was assessed with the Trail Making Tests (TMT) and the Color Word Interference Test (CWIT; both from the Delis-Kaplan Executive Function System, D-KEFS) [35]. The performance scores combined for this domain were condition 4 from the TMT (Number- 
Nakling et al.: Cognitive Deficits in Chronic Stroke Patients: Neuropsychological Assessment, Depression, and Self-Reports

Table 3. Overview over cognitive domains based on neuropsychological subtests (left column) and corresponding manuals for normative data and references (right column)

\begin{tabular}{|c|c|}
\hline Cognitive domains and included subtests & Test references for manuals and normative data \\
\hline \multicolumn{2}{|l|}{ General cognitive abilities } \\
\hline MMSE $^{1}$ & Folstein et al. [5] \\
\hline \multicolumn{2}{|l|}{ Visuoconstructive ability } \\
\hline Copy, RCFT & RCFT and Recognition Trial [38] \\
\hline \multicolumn{2}{|l|}{ Visual memory } \\
\hline \multicolumn{2}{|l|}{ Delayed recall, RCFT } \\
\hline \multicolumn{2}{|l|}{ Verbal memory } \\
\hline Total learning, CVLT II & CVLT II, Delis et al. [35] \\
\hline \multicolumn{2}{|l|}{$\begin{array}{l}\text { Short delay free recall, CVLT II } \\
\text { Long delay free recall, CVLT II }\end{array}$} \\
\hline \multicolumn{2}{|l|}{ Long delay free recall, CVLT II } \\
\hline \multicolumn{2}{|l|}{ Mental processing speed } \\
\hline Digit Symbol Test & WAIS IV [37] \\
\hline Color Naming, CWIT & D-KEFS [36] \\
\hline \multicolumn{2}{|l|}{ Number Sequencing, TMT } \\
\hline \multicolumn{2}{|l|}{ Letter Sequencing, TMT } \\
\hline \multicolumn{2}{|l|}{ Executive functioning } \\
\hline Inhibition, CWIT & D-KEFS [36] \\
\hline \multirow{2}{*}{\multicolumn{2}{|c|}{$\begin{array}{l}\text { Inhibition/Switching, CWIT } \\
\text { Number-Letter Switching, TMT }\end{array}$}} \\
\hline & \\
\hline \multicolumn{2}{|l|}{ Language production } \\
\hline Letter fluency & D-KEFS [36] \\
\hline Category fluency & \\
\hline
\end{tabular}

Letter Switching) and conditions 3 and 4 from the CWIT (Inhibition and Inhibition/Switching). Language production was assessed with the Verbal Fluency Tests (D-KEFS) including the performance scores from conditions 1 and 2 (Letter and Category Fluency, respectively). Processing speed included performance scores from the Digit Symbol Test (Wechsler Adult Intelligence Scale IV) [36], conditions 2 and 3 from the TMT (Number Sequencing and Letter Sequencing, respectively), and condition 1 from the CWIT (Color Naming). Two domains were assessed with one task: visual memory was assessed with the Rey Complex Figure Test (RCFT) [37] and solely the long delay recall score was included for this domain. Visuoconstructive ability was assessed with the copy subtest from the RCFT [37]. Neuropsychological examination was considered feasible when a patient was able to carry out at least 7 of the 15 subtests. Cronbach's $\alpha$ was calculated to examine the internal consistency for the cognitive domain scores except for abstract reasoning and visual memory since these domains were only assessed with one task. The analyses revealed that Cronbach's $\alpha$ varied between 0.78 and 0.93 , indicating acceptable levels of reliability.

Due to large age range in the sample (30-95 years) and well-known evidence for agerelated differences on cognitive performance score, raw scores of all individual neuropsychological tests were converted into standardized scores (mean $=50, \mathrm{SD}=10$ ) according to agecorrected original normative data from test manuals (as referenced in parentheses) for each test. Standardized scores for the verbal memory test also took gender into account. Table 3 
Nakling et al.: Cognitive Deficits in Chronic Stroke Patients: Neuropsychological Assessment, Depression, and Self-Reports

Table 4. Average cognitive performance scores for standardized scores on neuropsychological measures and proportion of impaired patients for the total sample and according to age groups ( $<65$ and $\geq 65$ years)

\begin{tabular}{|c|c|c|c|c|c|}
\hline & $\begin{array}{l}\text { Patients } \\
\text { assessed, } n\end{array}$ & $\begin{array}{l}\text { Total } \\
\text { sample }^{1}\end{array}$ & $\begin{array}{l}\text { Proportion } \\
\text { impaired of total } \\
\text { sample, \% }\end{array}$ & $\begin{array}{l}\text { Proportion } \\
\text { impaired <65 } \\
\text { years, \% }\end{array}$ & $\begin{array}{l}\text { Proportion } \\
\text { impaired } \geq 65 \\
\text { years, } \%\end{array}$ \\
\hline General cognitive abilities & & & 12 & 0 & 20 \\
\hline $\mathrm{MMSE}^{2}$ & 105 & $28.2(2.1)$ & & & \\
\hline Visuoconstructive ability & & & 19 & 8 & 27 \\
\hline Copy, RCFT & 96 & $46.7(15.2)$ & & & \\
\hline Visual memory & & & 34 & 26 & 40 \\
\hline Delayed recall, RCFT & 89 & $43.4(16.9)$ & & & \\
\hline Verbal memory & & & 13 & 0 & 21 \\
\hline Total learning, CVLT II & 100 & $48.7(11.4)$ & & & \\
\hline Short delay free recall, CVLT II & 99 & $47.3(12.0)$ & & & \\
\hline Long delay free recall, CVLT II & 99 & $48.5(10.9)$ & & & \\
\hline Mental processing speed & & & 20 & 20 & 21 \\
\hline Digit Symbol Test (WAIS IV) & 100 & 41.7 (7.9) & & & \\
\hline Color Naming, CWIT & 104 & $40.5(10.2)$ & & & \\
\hline Number Sequencing, TMT & 105 & 40.7 (11.9) & & & \\
\hline Letter Sequencing, TMT & 103 & $41.6(12.2)$ & & & \\
\hline Executive functioning & & & 26 & 10 & 37 \\
\hline Inhibition, CWIT & 99 & $45.2(11.7)$ & & & \\
\hline Inhibition/Switching, CWIT & 99 & $42.0(12.8)$ & & & \\
\hline Number-Letter Switching, TMT & 103 & $37.0(14.1)$ & & & \\
\hline Language production & & & 12 & 5 & 17 \\
\hline Letter fluency & 99 & $48.6(12.4)$ & & & \\
\hline Category fluency & 98 & 52.3 (12.9) & & & \\
\hline
\end{tabular}

MMSE, Mini-Mental State Examination; RCFT, Rey Complex Figure Test; CVLT II, California Verbal Learning Test II; WAIS IV, Wechsler Adult Intelligence Scale IV; CWIT, Color Word Interference Test; TMT, Trail Making Test. ${ }^{1}$ Values represent mean (standard deviation). ${ }^{2}$ Raw score.

gives an overview over cognitive domains and corresponding subtests as well as references for manuals for normative data.

For analyses, cognitive impairment was defined as a performance of at least $-1.5 \mathrm{SD}$ for each domain based on the reference distribution of the normative sample. This cutoff is frequently chosen in aging studies and derived from findings on mild cognitive impairment, a risk factor for dementia [38, 39]. Mild cognitive impairment is defined as lower performance in one or more cognitive domains as would be expected for the patient's age. Patients with mild cognitive impairment frequently demonstrate problems performing complex functional tasks, need more time, are less efficient, and make more errors. They may, however, still maintain their independence of function in daily life. Table 4 demonstrates performance of the sample on individual cognitive tests.

\section{Emotional Functioning}

The Hospital Anxiety and Depression Scale (HADS) [40] was included to assess depression and anxiety. The patient has to answer seven items for each subscale on a 4-point response scale (0-3), with possible scores ranging from 0 to 21 . The HADS has been validated in stroke patients [41]. Scores ranging from 0 to 7 for either subscale are regarded as being within the 
Nakling et al.: Cognitive Deficits in Chronic Stroke Patients: Neuropsychological Assessment, Depression, and Self-Reports

normal range. Since best sensitivity and specificity (sensitivity: 0.72 and 0.78 and specificity 0.86 and 0.74 for depression and anxiety, respectively) have been shown using subscale scores $>7$. This was applied to our data to indicate depression/anxiety [40].

\section{Stroke Impairment Scale}

The SIS (version 3) was developed to take the patients' and caregivers' perspective of impact of stroke on health and functional status into account $[42,43]$. The SIS includes 59 items on 8 subscales including strength, hand function, basic and instrumental activities of daily living, mobility and communication, emotion, memory and thinking, and participation. For this study, the mtSIS, comprising seven items, was used to assess self-reported cognitive function. Four items address memory function (e.g., "In the past week how difficult was it for you to remember things that people just told you?"). Concentration, mental processing speed, and problem solving are addressed with one item each. Items are scored on a 5-point scale ( 1 = extremely difficult, 5 = not difficult at all). Raw scores are transformed according to the manual and a score of 100 indicates no self-reported cognitive impairment.

\section{Statistics}

Descriptive statistics were used to describe the sample characteristics. In a first step, we examined differences on baseline characteristics between patients returning for assessment and those not returning using $\chi^{2}$ tests for categorical variables and $t$ tests for numerical variables. The frequency of cognitive impairment in each of the 6 cognitive domains was calculated. Since the cognitive impairment seems crucial in aging individuals due to the risk of further decline, the proportion of patients with and without impairment was compared between age groups below 65 and $\geq 65$ years. This cutoff was based on the literature indicating that cognitive decline increases at about the age of 65 years [44].

Pearson correlations were used to examine the relationship between cognitive measures and symptoms of depression and anxiety. A linear regression was run to determine if demographic (gender, age) and cognitive measures predicted depression. In a final step, Pearson correlations were used to examine the relationship between cognitive measures and selfreported cognitive function on the SIS subscale before running logistic regression models to examine the predictive value of self-reported cognitive function (according to the mtSIS) for cognitive measures. For the last, cognitive domain variables were coded dichotomous, i.e., as being within the normal range versus being impaired (1.5 SD below normative data). Reporting cognitive problems or not, i.e., an mtSIS score of 100 (no reported impairment) was included as a covariate. Sensitivity (the instruments' ability to correctly detect patients who do have a specific condition, i.e., cognitive impairment) and specificity (the instruments' ability to detect patients without a condition), positive predictive values (probability that patients with a positive test truly have an impairment), and the area under the receiving operating characteristic curve (AUC) of the mtSIS were calculated for each of the cognitive performance measures. For all analyses, $p$ values $<0.05$ are reported and considered statistically significant. No formal adjustment for multiple comparisons is carried out due to the exploratory nature of the analyses.

\section{Results}

Demographic Characteristics for Returning and Not Returning Patients

Upon admission, $n=322$ patients were registered. The average age for the total sample was 71.5 years (SD 14.4); $55 \%$ of the patients were male. Eighty-nine percent had an ischemic and $11 \%$ a hemorrhagic stroke. Of the originally registered patients, $44 \%$ returned after 1 
Fig. 1. Frequency of cognitive impairment in chronic stroke patients.

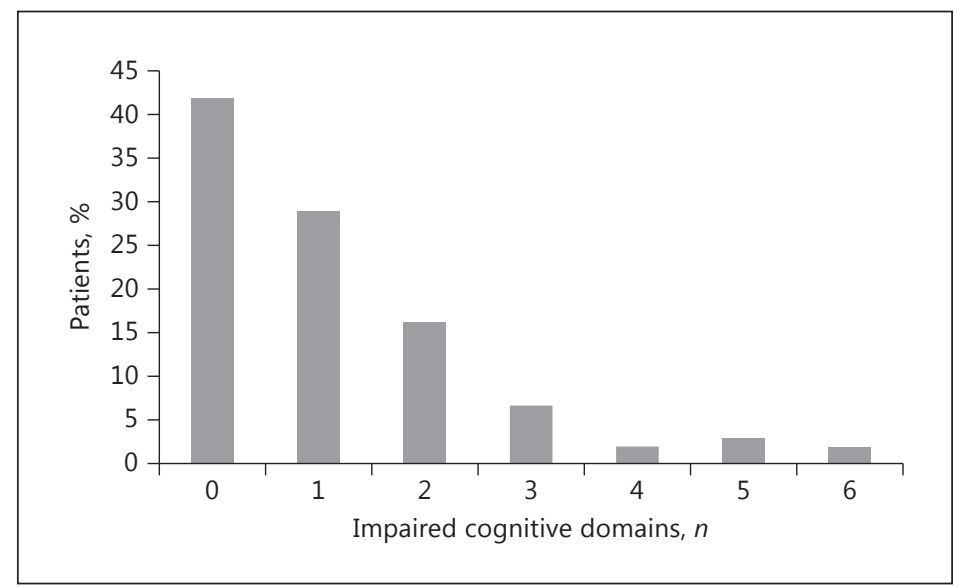

year. Of these $n=143, n=105$ were available for neuropsychological assessment (Table 1). Patients not returning were significantly older compared to those who underwent neuropsychological assessment and who were included in this study (years of age of study sample vs. not returning patients: mean 67.5 years [SD 12.5] vs. mean 75.7 years [SD 13.8]; $p<0.001$ ), had a higher NIHSS upon admission (NIHSS study sample vs. not returning patients: mean 4.9 [SD 4.6] vs. mean 6.4 [SD 5.5]; $p<0.001$ ), indicating a lower function in the patients not returning, and had significantly more often peripheral artery disease (study sample vs. not returning patients $12.5 \%$ vs. $8.9 \%, p<0.001$ ). None of the other registered variables showed significant differences (see Table 1 for characteristics of the total sample, returning and not returning patients).

\section{Cognitive Performance}

There was some variation in the number of patients who completed individual tests since some patients indicated increasing fatigue, discontinued assessment, or had motor deficits (Table 3). Of the 105 patients assessed, 58\% revealed cognitive impairment (Fig. 1) reaching from isolated impairment in 1 domain (29\%) to impairment in all 6 domains (2\%). Cognitive deficits for the domain of executive functioning occurred significantly more frequently in older patients $\left(\chi^{2}=8.89, p=0.003\right)$. Only individuals in the age group $\geq 65$ years showed impairment for the MMSE and the verbal memory domain. There were no gender differences for cognitive impairment. Based on the neuropsychological assessment, the highest proportion of impairment was found with measures of visual memory, executive function, and processing speed.

\section{Emotional Functioning}

Eighty-eight patients answered the HADS. Average scores for both anxiety (mean $=3.2$, SD 3.3) and depression (mean $=3.1$, SD 3.2) scales did not indicate considerable emotional symptoms on a group level. Further analyses indicated that 12 (13.6\%) patients reported elevated scores (>7) on the anxiety scale and $11(12.5 \%)$ on the depression scale. Neither age, nor gender, nor MMSE was significantly correlated with either depression or anxiety. With regard to cognitive measures, there were significant correlations between depression scores and executive functioning, processing speed, and language production $(r=-0.31, r=-0.36$, $r=-0.37$; all $p \leq 0.001$ ). A hierarchical regression analysis, including demographic variables (age, gender) and performance scores on the cognitive domains that were significantly correlated with symptoms of depression, revealed that age and gender were no significant 
Nakling et al.: Cognitive Deficits in Chronic Stroke Patients: Neuropsychological Assessment, Depression, and Self-Reports

Table 5. Results of the hierarchical regression analysis predicting depression

\begin{tabular}{|c|c|c|c|c|c|c|}
\hline & & $R^{2}$ & B & SE B & $\beta$ & $t$ \\
\hline \multirow[t]{3}{*}{ Step 1} & Constant & \multirow{3}{*}{0.2} & 11.23 & 3.14 & & 3.57 \\
\hline & Age & & -0.03 & 0.03 & -0.10 & -0.82 \\
\hline & Gender & & -0.40 & 0.77 & 0.06 & -0.52 \\
\hline \multirow[t]{3}{*}{ Step 2} & Language production & \multirow{3}{*}{$0.18^{*}$} & 0.11 & 0.05 & -0.36 & -2.41 \\
\hline & Executive function & & -0.12 & 0.17 & -0.12 & -0.74 \\
\hline & Processing speed & & 0.02 & 0.05 & 0.06 & 0.44 \\
\hline
\end{tabular}

Table 6. Results of the sensitivity and specificity analysis of mtSIS subscale scores predicting deficits in several cognitive domains

\begin{tabular}{lllll}
\hline & Sensitivity & Specificity & PPV & AUC \\
\hline Language production & 0.19 & 0.39 & 0.13 & 0.46 \\
Executive function & 0.40 & 0.42 & 0.33 & 0.38 \\
Processing speed & 0.35 & 0.40 & 0.26 & 0.41 \\
Visual memory & 0.39 & 0.36 & 0.26 & 0.54 \\
Visuoconstructive ability & 0.27 & 0.39 & 0.18 & 0.49 \\
Verbal memory & 0.21 & 0.37 & 0.13 & 0.47 \\
Global cognitive function (MMSE) & 0.21 & 0.38 & 0.14 & 0.41 \\
\hline
\end{tabular}

PPV, positive predictive value; AUC, area under the curve; MMSE, Mini-Mental State Examination.

predictors for depression. Including cognitive domain scores in addition to demographic variables into the model increased $R^{2}$ from 2 to $18 \%(p=0.008)$. Additional analyses revealed that language production contributed most to model prediction (Table 5). The Variance Inflation Factor (VIF) was close to 1, indicating that collinearity was not a problem for this model.

\section{Association between Neuropsychological Performance and Self-Reported Function}

In total, 88 patients answered the SIS. The median for the mtSIS was 93.75, indicating that a considerable number of patients in this sample hardly reported any problems. Thirty-two patients (36\%) reported that they had not experienced any cognitive problems at all during the past week. Gender analyses indicated a significant difference in that women reported more problems compared to men $(t=2.20 ; p=0.0340)$. There were significant correlations between the mtSIS and verbal memory $(r=0.26, p=0.017)$, executive functioning $(\mathrm{r}=0.27$, $p=0.010)$, and language production $(r=0.35, p<0.001)$. There was a significant negative correlation between mtSIS and the total number of impaired domains $(r=-0.34, p<0.001)$. The mtSIS did not correlate with HADS depression or anxiety scales.

Results of the logistic regression analyses with the cognitive measures as dependent variables are shown in Table 5. The mtSIS was not shown to be a significant predictor in any of the models. Sensitivity and specificity were $<0.5$ for all cognitive domains with the highest sensitivity, specificity, and positive predictive value for the executive domain (Table 6). AUC was largest for the visuoconstructive ability (AUC 0.54). 


\section{Discussion}

The results revealed cognitive impairment in approximately $60 \%$ of chronic stroke patients when assessed with neuropsychological methods compared to $12 \%$ based on the MMSE. Patients aged 65 years or older demonstrated significantly more impairment in executive functioning and verbal memory compared to those aged $<65$ years. Symptoms of depression correlated significantly with 3 cognitive domains, and linear regression indicated a significant increase in explained variance when cognitive domains were included in the model. Lastly, we found significant correlations between the SIS memory and thinking subscale and cognitive measures. Yet, the final analyses showed that sensitivity for the SIS subscale was low for all cognitive domains with positive predictive values all well below 0.5.

In general, the results showing persistent cognitive impairment in chronic stroke patients were in accordance with earlier findings $[8,9,11,12]$. In our sample, this meant for the majority of patients impairment in $1(29 \%)$ or 2 domains (16\%). Only $12 \%$ of these cognitively impaired patients would have been correctly classified as such when using the MMSE score alone.

The proportion of impairment found in our study was higher than in an earlier study [9] but lower than previously reported [8]. Likewise, numbers were varying with regard to the most frequently impaired domain. In our sample, the visual memory domain was most frequently impaired, while others reported impaired attention [8] or abstract reasoning [9] as the domain with the most frequent impairment. The deviating percentages of impairment and their prevalence in cognitive domains may be explained by the choice of instruments used for assessment and sample characteristics such as age, severity of stroke, or point of time of assessment.

In our sample, the severity of stroke as indicated by the rather low average NIHSS scores indicates that the majority of patients had rather a mild stroke. Moreover, the additional analyses revealed that the patients returning (compared to those not returning) had milder strokes (according to the NIHSS upon admission) and were younger. In addition, 38 of those who were assessed were either excluded due to aphasia or were unable to complete the test battery. Thus, several of these patients may have been cognitively impaired. This limits the generalizability of our findings and indicates that our estimates of cognitive impairment are an underestimate. Nevertheless, we find the results important as they indicate that even patients with rather mild strokes demonstrate persistent cognitive impairment. Furthermore, we did not set an age cutoff, although we were aware that age by itself is associated with cognitive decline. Our sample included 6 patients $\geq 85$ years. Rerunning analyses and excluding patients above the age of 85 years did not change any of the presented results. Thus, we felt it was correct to include them as they were willing to undergo comprehensive assessment. In fact, 2 of them did not show impairment in any domains, and the oldest patient showed impairment in only 1 cognitive domain.

The choice of the Rey Complex Figure Test (RCFT) may have contributed to findings indicating a high impairment in the visual memory domain. The test requires visuomotor abilities during the copying condition (the score used to represent the visuoconstructive domain) and subsequently reproducing/memorizing a complex line drawing (visual memory domain). Thus, failure may arise from constructional disability, impaired visual or spatial memory or an interaction of both, or even other factors [45]. Through this complexity, the RCFT may therefore tap slightly different abilities than a visual memory test where items must just be remembered. Barker-Collo et al. [15] including the RCFT in a 5-year follow-up study found that visual memory scores were within 1 SD of the normative data whereas the group average copy function was clearly impaired being $>2$ SD below normative data. They suggest that the impaired copy score relates to executive functions which are required when copying the 
Nakling et al.: Cognitive Deficits in Chronic Stroke Patients: Neuropsychological Assessment, Depression, and Self-Reports

figure. In our patients, $19 \%$ showed impairment in the copy subtest. Of these, $63 \%$ also showed impairment in the long delayed recall subtest, whereas only $24 \%$ of those performing within the normal range in the copy subtest showed impairment in the long delay recall. Since we included only one visuoconstructive test, it is not possible to untangle whether motor deficits, executive functions, or both as suggested by Barker-Collo et al. [15] contributed to the visual memory impairment. Our data suggest, however, that patients impaired during copying were frequently also impaired in visual memory function.

The findings showing impairment in executive functioning and processing speed are in general accordance with earlier findings $[9,10,12]$. In particular, the finding that executive impairment occurred more frequently in the older age group is notable. It has been emphasized that executive dysfunction and reduced processing speed frequently occur in individuals with vascular cognitive impairment [46] who may show further cognitive decline and progression to dementia [12]. We can only speculate that this applies to some of the patients in our study. Yet, it highlights the need for longitudinal studies including comprehensive cognitive assessment in stroke patients. This importance is further stressed and extended by the results showing significant correlations between the symptoms of depression, executive function, processing speed, and language production.

The proportion of patients reporting symptoms of depression and anxiety in our study (13-14\%, respectively) was considerably lower compared to those reported by Ayerbe et al. [47] (28\% depression and 33\% anxiety) or Kauhanen et al. [23] (42\%) and more similar to those of Astrom et al. [48] (16\% depression). Yet, Ayerbe et al. [47] found that cognitive impairment (based on the MMSE) predicted depression throughout a 5-year follow-up and showed that prevalence of depression even doubles in these individuals. Our regression model revealed that language production showed the strongest inverse relationship with depression. That is to say, reduced word production indicated more symptoms of depression. Despite requiring semantic knowledge and processing, these tests, in particular letter fluency, have been shown to tap a high degree of executive functioning since retrieval strategies must be initiated, given responses must be kept in mind to avoid repetitions (working memory), and semantically close words must be avoided [49]. Executive dysfunction has earlier been shown to be closely associated with activities of daily living [50] and accelerated cognitive decline [16]. The clear link between depression and executive functioning stresses the importance to follow up these patients to prevent them to end up in a vicious circle.

The final analyses showing that the mtSIS subscale correlated significantly with cognitive domains of language, executive function, and memory are new and noteworthy. To the best of our knowledge, no earlier study has combined the mtSIS with neuropsychological assessment. At first it has to be acknowledged that self-reported functioning based on the $\mathrm{mtSIS}$ was rather positive as indicated by a high median. This seems to be in some contrast to earlier studies demonstrating cognitive complaints in more than $50 \%$ of stroke patients [28]. Duncan et al. [43] found that items were too easy to answer and would only detect difficulties in severely impaired stroke patients. We found significant positive correlations between $\mathrm{mtSIS}$ and executive functioning and language production, even though 4 of the 7 questions from the mtSIS relate to memory function. This indicates that patients reporting good cognitive functioning also perform equally well. However, the sensitivity and specificity for the $\mathrm{mtSIS}$ regarding all cognitive domains were critically low. Although the missing empirical evidence between objective and self-reported cognitive function is not a new phenomenom, this indicates that clinicians have to be cautious using the mtSIS to identify patients with cognitive impairment. As mentioned above, our sample contained patients with rather mild strokes and the majority of patients showed impairment in 1 or 2 cognitive domains. Thus, impairment may not have been as prominent as necessary to be mentioned. In fact, we found a significant correlation between mtSIS scores and the number of impaired domains. This 
Nakling et al.: Cognitive Deficits in Chronic Stroke Patients: Neuropsychological Assessment, Depression, and Self-Reports

could imply that a certain "threshold" of impairment must be passed before it affects routine daily activities often enough to be noted and reported by the patient. Yet, the problem of sensitivity has been commented on by Duncan et al. [43] even in a more heterogeneous group of patients. In their review regarding subjective cognitive complaints, van Rijsbergen et al. [29] underlined the urgent need for further insight in poststroke complaints and their multifaceted underlying reasons.

The current study has a number of weaknesses. Besides the already mentioned shortcomings, the study had a relatively small sample size. This does not affect the primary messages of the results. However, a larger sample would have enabled us to analyze cognitive impairment based on stroke subtypes or medical factors, and the includion of more severely affected patients would have increased the generalizability of findings. We did not exclude patients with a recurrent stroke or earlier transient ischemic attack although being aware that their cognitive performance may have been influenced by these factors. As earlier indicated the sample in this study suffers from attrition bias since only $44 \%$ of the originally registered patients returned. Analyses revealed that further reference bias is possible since the study includes individuals with rather mild stroke (as indicated by the NIHSS) and individuals who are able to undergo neuropsychological assessment. Thus, patients with more severe stroke and more severe cognitive impairment and/or depression are underrepresented. Additionally, selection bias may have occurred due to the inclusion and exclusion criteria. Finally, statistically significant results need to be interpreted with caution as no multiplicity adjustment was used.

\section{Conclusion}

Cognitive impairment in chronic stroke patients occurs frequently and persistently within the first year after stroke. It is important to provide more comprehensive neuropsychological assessment besides screening measures to detect cognitive deficits as well as their interaction with depressive symptoms. The findings underline the fact that stroke patients should be monitored and that longitudinal data are required. Providing more comprehensive assessment to stroke patients enables clinicians to offer adequate treatment and to define individuals needing further attendance. Clinicians should be aware of possible impairment even though patients are not reporting them.

\section{Disclosure Statement}

The authors declare that they have no competing interests.

\section{Funding Sources}

This work was supported by a grant from the Kavli Research Center for Aging and Dementia to Eike Wehling and a grant from the Research Council of Norway to Håkon Hofstad. 
Nakling et al.: Cognitive Deficits in Chronic Stroke Patients: Neuropsychological Assessment, Depression, and Self-Reports

\section{References}

1 Lopez AD, Mathers CD, Ezzati M, Jamison DT, Murray CJ: Global and regional burden of disease and risk factors, 2001: systematic analysis of population health data. Lancet 2006;367:1747-1757.

-2 Donnan GA, Fisher M, Macleod M, Davis SM: Stroke. Lancet 2008;371:1612-1623.

3 Kinsella K: Urban and rural dimensions of global population aging: an overview. J Rural Health 2001;17:314322.

-4 Pendlebury ST, Rothwell PM: Risk of recurrent stroke, other vascular events and dementia after transient ischaemic attack and stroke. Cerebrovasc Dis 2009;27(suppl 3):1-11.

5 Folstein MF, Folstein SE, McHugh PR: "Mini-mental state." A practical method for grading the cognitive state of patients for the clinician. J Psychiatr Res 1975;12:189-198.

6 Nasreddine ZS, Phillips NA, Bedirian V, Charbonneau S, Whitehead V, Collin I, Cummings JL, Chertkow H: The Montreal Cognitive Assessment, MoCA: a brief screening tool for mild cognitive impairment. J Am Geriatr Soc 2005;53:695-699.

7 Nys GM, van Zandvoort MJ, de Kort PL, Jansen BP, Kappelle LJ, de Haan EH: Restrictions of the Mini-Mental State Examination in acute stroke. Arch Clin Neuropsychol 2005;20:623-629.

8 Lesniak M, Bak T, Czepiel W, Seniow J, Czlonkowska A: Frequency and prognostic value of cognitive disorders in stroke patients. Dement Geriatr Cogn Disord 2008;26:356-363.

-9 Nys GM, van Zandvoort MJ, de Kort PL, van der Worp HB, Jansen BP, Algra A, de Haan EH, Kappelle LJ: The prognostic value of domain-specific cognitive abilities in acute first-ever stroke. Neurology 2005;64:821-827.

10 Stephens S, Kenny RA, Rowan E, Allan L, Kalaria RN, Bradbury M, Ballard CG: Neuropsychological characteristics of mild vascular cognitive impairment and dementia after stroke. Int J Geriatr Psychiatry 2004;19:10531057.

11 Hochstenbach JB, den Otter R, Mulder TW: Cognitive recovery after stroke: a 2-year follow-up. Arch Phys Med Rehabil 2003;84:1499-1504.

12 Sachdev PS, Chen X, Brodaty H, Thompson C, Altendorf A, Wen W: The determinants and longitudinal course of post-stroke mild cognitive impairment. J Int Neuropsychol Soc 2009;15:915-923.

13 del Ser T, Barba R, Morin MM, Domingo J, Cemillan C, Pondal M, Vivancos J: Evolution of cognitive impairment after stroke and risk factors for delayed progression. Stroke 2005;36:2670-2675.

14 Ballard C, Rowan E, Stephens S, Kalaria R, Kenny RA: Prospective follow-up study between 3 and 15 months after stroke: improvements and decline in cognitive function among dementia-free stroke survivors $>75$ years of age. Stroke 2003;34:2440-2444.

15 Barker-Collo S, Feigin VL, Parag V, Lawes CM, Senior H: Auckland Stroke Outcomes Study. Part 2. Cognition and functional outcomes 5 years poststroke. Neurology 2010;75:1608-1616.

$\checkmark 16$ Levine DA, Galecki AT, Langa KM, Unverzagt FW, Kabeto MU, Giordani B, Wadley VG: Trajectory of cognitive decline after incident stroke. JAMA 2015;314:41-51.

17 Hosking S, Marsh N, Friedman P: Depression at 3-months poststroke in the elderly: predictors and indicators of prevalence. Aging Neuropsychol Cogn 2000; 7:205-216.

18 Ayerbe L, Ayis S, Crichton S, Wolfe CD, Rudd AG: The natural history of depression up to 15 years after stroke: the South London Stroke Register. Stroke 2013;44:1105-1110.

19 Pan A, Sun Q, Okereke OI, Rexrode KM, Hu FB: Depression and risk of stroke morbidity and mortality: a metaanalysis and systematic review. JAMA 2011;306:1241-1249.

20 Chemerinski E, Robinson RG, Kosier JT: Improved recovery in activities of daily living associated with remission of poststroke depression. Stroke 2001;32:113-117.

-21 Sturm JW, Donnan GA, Dewey HM, Macdonell RA, Gilligan AK, Srikanth V, Thrift AG: Quality of life after stroke: the North East Melbourne Stroke Incidence Study (NEMESIS). Stroke 2004;35:2340-2345.

-22 Barker-Collo SL: Depression and anxiety 3 months post stroke: prevalence and correlates. Arch Clin Neuropsychol 2007;22:519-531.

23 Kauhanen M, Korpelainen JT, Hiltunen P, Brusin E, Mononen H, Maatta R, Nieminen P, Sotaniemi KA, Myllyla VV: Poststroke depression correlates with cognitive impairment and neurological deficits. Stroke 1999;30: 1875-1880.

24 Nys GM, van Zandvoort MJ, van der Worp HB, de Haan EH, de Kort PL, Kappelle LJ: Early depressive symptoms after stroke: neuropsychological correlates and lesion characteristics. J Neurol Sci 2005;228:27-33.

-25 Hackett ML, Yapa C, Parag V, Anderson CS: Frequency of depression after stroke: a systematic review of observational studies. Stroke 2005;36:1330-1340.

26 Murata Y, Kimura M, Robinson RG: Does cognitive impairment cause post-stroke depression? Am J Geriatr Psychiatry 2000;8:310-317.

27 Duits A, Munnecom T, van Heugten C, van Oostenbrugge RJ: Cognitive complaints in the early phase after stroke are not indicative of cognitive impairment. J Neurol Neurosurg Psychiatry 2008;79:143-146.

28 Hochstenbach J, Prigatano G, Mulder T: Patients' and relatives' reports of disturbances 9 months after stroke: subjective changes in physical functioning, cognition, emotion, and behavior. Arch Phys Med Rehabil 2005;86: $1587-1593$

29 van Rijsbergen MW, Mark RE, de Kort PL, Sitskoorn MM: Subjective cognitive complaints after stroke: a systematic review. J Stroke Cerebrovasc Dis 2014;23:408-420. 
-30 Toomela A, Pulver A, Tomberg T, Orasson A, Tikk A, Asser T: Possible interpretation of subjective complaints in patients with spontaneous subarachnoid haemorrhage. J Rehabil Med 2004;36:63-69.

-31 Passier PE, Visser-Meily JM, van Zandvoort MJ, Post MW, Rinkel GJ, van Heugten C: Prevalence and determinants of cognitive complaints after aneurysmal subarachnoid hemorrhage. Cerebrovasc Dis 2010;29:557563.

-32 Lamb F, Anderson J, Saling M, Dewey H: Predictors of subjective cognitive complaint in postacute older adult stroke patients. Arch Physical Med Rehabil 2013;94:1747-1752.

-33 Adams HP Jr, Bendixen BH, Kappelle LJ, Biller J, Love BB, Gordon DL, Marsh EE 3rd: Classification of subtype of acute ischemic stroke. Definitions for use in a multicenter clinical trial. TOAST. Trial of Org 10172 in Acute Stroke Treatment. Stroke 1993;24:35-41.

34 Delis DC, Kramer JH, Kaplan E, Ober BA: California Verbal Learning Test II (CVLT II). Stockholm, Pearson Assessment, 2004.

35 Delis DC, Kaplan E, Kramer JH: Delis-Kaplan Executive Function System (D-KEFS). San Antonio, Psychological Corporation, 2001.

36 Wechsler D: Wechsler Adult Intelligence Scale - Fourth Edition (WAIS IV). New York, The Psychological Corporation, 2008.

37 Meyers JE, Meyers KR: Rey Complex Figure Test and Recognition Trial (RCFT). Lutz, Psychological Assessment Resources, 1995.

-38 Petersen RC, Smith GE, Waring SC, Ivnik RJ, Tangalos EG, Kokmen E: Mild cognitive impairment: clinical characterization and outcome. Arch Neurol 1999;56:303-308.

39 Tuokko HA, Hultsch DF: Mild Cognitive Impairment - International Perspectives. Hove, Taylor \& Francis, 2015.

40 Brennan C, Worrall-Davies A, McMillan D, Gilbody S, House A: The Hospital Anxiety and Depression Scale: a diagnostic meta-analysis of case-finding ability. J Psychosom Res 2010;69:371-378.

41 Aben I, Verhey F, Lousberg R, Lodder J, Honig A: Validity of the beck depression inventory, hospital anxiety and depression scale, SCL-90, and hamilton depression rating scale as screening instruments for depression in stroke patients. Psychosomatics 2002;43:386-393.

42 Duncan PW, Wallace D, Lai SM, Johnson D, Embretson S, Laster LJ: The stroke impact scale version 2.0. Evaluation of reliability, validity, and sensitivity to change. Stroke 1999;30:2131-2140.

-43 Duncan PW, Bode RK, Min Lai S, Perera S; Glycine Antagonist in Neuroprotection Americans Investigators: Rasch analysis of a new stroke-specific outcome scale: the Stroke Impact Scale. Arch Phys Med Rehabil 2003; 84:950-963.

44 Hedden T, Gabrieli JD: Insights into the ageing mind: a view from cognitive neuroscience. Nat Rev Neurosci 2004;5:87-96.

45 Lezak MD, Howieson DB, Loring DW: Neuropsychological Assessment, ed 4. Oxford, Oxford University Press, 2004.

46 Nordlund A, Rolstad S, Klang O, Lind K, Hansen S, Wallin A: Cognitive profiles of mild cognitive impairment with and without vascular disease. Neuropsychology 2007;21:706-712.

47 Ayerbe L, Ayis S, Rudd AG, Heuschmann PU, Wolfe CD: Natural history, predictors, and associations of depression 5 years after stroke: the South London Stroke Register. Stroke 2011;42:1907-1911.

48 Astrom M, Adolfsson R, Asplund K: Major depression in stroke patients. A 3-year longitudinal study. Stroke 1993;24:976-982.

49 Luo L, Luk G, Bialystok E: Effect of language proficiency and executive control on verbal fluency performance in bilinguals. Cognition 2010;114:29-41.

50 Pohjasvaara T, Leskela M, Vataja R, Kalska H, Ylikoski R, Hietanen M, Leppavuori A, Kaste M, Erkinjuntti T: Post-stroke depression, executive dysfunction and functional outcome. Eur J Neurol 2002;9:269-275. 